\title{
Revealing the polarity of actin filaments by cryo-electron tomography
}

2

3 Bruno Martins ${ }^{1}$, Simona Sorrentino ${ }^{1}$, Wen-Lu Chung ${ }^{1}$, Meltem Tatli ${ }^{1}$, Ohad Medalia ${ }^{1^{*}}$, and $4 \quad$ Matthias Eibauer ${ }^{1 *}$

5

6

7

8

9

10

11

12

13

14

15

16

17 (m.eibauer@bioc.uzh.ch)

18

19

20 


\section{Summary}

22 The actin cytoskeleton plays a fundamental role in numerous cellular processes, such as cell

23 motility, cytokinesis, and adhesion to the extracellular matrix. Revealing the polarity of

24 individual actin filaments in cells, would foster an unprecedented understanding of cytoskeletal 25 processes and their associated mechanical forces. Cryo-electron tomography provides the 26 means for high-resolution structural imaging of cells. However, the low signal-to-noise ratio of 27 cryo-tomograms obscures the high frequencies and therefore the polarity of actin filaments 28 cannot be directly measured. Here, we developed an approach that enables to determine the 29 polarity of actin filaments in cellular cryo-tomograms. We applied it to reveal the actin polarity 30 distribution in focal adhesions, and show a linear relation between actin polarity and distance 31 from the apical boundary of the adhesion site. 


\section{Introduction}

Actin polymerization drives cell motility and is a central factor in mediating contractile forces in cells (Holmes et al., 1990; Merino et al., 2018; Pollard and Borisy, 2003). Actin filaments (Egelman et al., 1982; Galkin et al., 2015) assemble into complex networks (Malik-Garbi et al., 2019; Xu et al., 2012), which are essential for their activity in the cell. Reconstructing individual actin filaments at sub-nanometer resolution inside cells, would provide an unparalleled view on actin networks, and would allow a more fine-grained modelling of cytoskeletal based mechanical processes (Hervas-Raluy et al., 2019).

A prominent mechanosensitive process, involving actomyosin contractility, occurs at the integrin-based interaction sites of cells with the extracellular matrix (Burridge and Guilluy, 2016; Geiger et al., 2009). These interactions are mediated by adhesive structures such as focal adhesions (FAs) (Legate et al., 2011; Shemesh et al., 2005; Zaidel-Bar et al., 2007). The organization of proteins and the role of the actin network in FAs has been intensively studied using fluorescent and electron microscopy (Kanchanawong et al., 2010; Patla et al., 2010), however, the 3D architecture of FAs, including the polarity and position of each individual actin filament was not yet shown.

Cryo-electron tomography (cryo-ET) allows to reconstruct 3D density maps of unperturbed cells at a resolution of 2-3 nm (Beck and Baumeister, 2016; Lucic et al., 2005; Weber et al., 2019). Therefore, single actin filaments can readily be detected in tomograms of eukaryotic cells (Jasnin et al., 2019; Medalia et al., 2002). Moreover, using direct electron detectors permit to recognize the characteristic helical shape of $\mathrm{F}$-actin that is in clear distinction to other cellular filaments. However, cryo-tomograms suffer from a low signal-to-noise ratio (SNR) (Forster et al., 2008; Pei et al., 2016), and from missing information due to the limited tilt range during data acquisition, referred to as the missing wedge (Lucic et al., 2005). Subtomogram averaging can compensate for these issues (Beck and Baumeister, 2016; Forster et al., 2005; Himes and Zhang, 2018; Schur et al., 2016), but it is computationally more demanding and is lacking the robustness and level of standardization as the procedures used for data analysis in single particle cryo-electron microscopy (Nogales, 2016; Scheres, 2012). 
Here we developed a set of MATLAB scripts that enable, in conjunction with RELION (Scheres, 2012), the $3 D$ reconstruction of actin filaments from cryo-tomograms. It is based on transforming subtomogram averaging into a single particle task, and the determination of their polarity is built on a robust statistical analysis of the individual filaments. Furthermore, this actin polarity toolbox (APT) features tools for spatial and topological analysis of the reconstructed actin filament networks and their visualization.

Using a correlative fluorescence microscopy and cryo-ET approach (Elad et al., 2013; Patla et al., 2010; Sartori et al., 2007), we unveil the 3D architecture of the actin cytoskeleton at FAs. We show that the actin polarity distribution correlates with the position along the FA and that regions of mixed polarity are concentrated at the periphery of the characteristic actin bundles.

\section{Results}

Determining actin polarity by APT. The first aim of APT is the reconstruction of an actin filament at a resolution that allows the unambiguous determination of the filament polarity (better than $20 \AA$ ). Therefore, APT requires cryo-tomograms and segmentations of the actin cytoskeleton as input data (Fig. 1a). Subsequently, the segmented filaments are subdivided into segments with equidistant spacing. We make use of the observation that a projection of a subtomogram along the electron beam axis is approximately invariant of the missing wedge orientation and can be treated as a single particle image (Supplemental Fig. 1). Thus, single particle image processing packages, such as cryoSPARC (Punjani et al., 2017) or RELION (Scheres, 2012), can be used to reconstruct the actin filament from the segments. Interestingly, this approach is not only restricted to filamentous structures (Supplemental Fig. 2).

Our main goal is to determine the polarity of all the initially segmented filaments. Therefore, based on the filament average, APT calculates for each segment the position of its plus-end in the cryo-tomograms (Supplemental Fig. 3a). During the 3D reconstruction step, APT obscures the correlation between segments and filaments, thus for each filament multiple independent polarity observations can be statistically evaluated, which greatly reduces the error of polarity determination, as shown in the validation of the method (see below). 
The segmentations can be conducted on contrast enhanced tomograms in order to facilitate a better detection of actin filaments. We do not assume the segmentations to be free of false positives, because they will be efficiently sorted out at a later stage. However, we assume that each filament or detected part is represented by a unique set of voxels, therefore filaments should not touch each other in the segmentations. The fact that a filament may appear divided into parts, for instance when it runs through low-contrast, dense or crowded regions, where it cannot be tracked unambiguously, has no detrimental effect on its polarity determination, but leads to an underestimation of the actual filament length.

The workflow of APT is shown in Fig. 1a. A detailed description of the consecutive steps can be found in the Method Details section.

Validation of APT. The missing wedge affects the reconstruction of a tomogram in an anisotropic manner (Lucic et al., 2005). As a consequence, filaments running parallel to the tilt-axis are better resolved than filaments oriented orthogonal to the tilt-axis (Supplemental Fig. 1). It is fundamental to ensure that our projection approach for subtomogram averaging is not biased by the missing wedge. Additionally, the impact of SNR and polarity distribution on the output precision of APT should be verified.

For validation purposes we implemented a ground truth data generator (see Method Details), that creates modelled tomograms of actin bundles (volume $353 \times 353 \times 353 \mathrm{~nm}^{3}$ with a pixelsize of $3.44 \AA$ ), utilizing EMD-6179 (Galkin et al., 2015) as filament density, with three adjustable parameters: the angle $\varphi$ of the bundle with the tilt-axis, the SNR of the tomogram (Forster et al., 2008; Pei et al., 2016), and the polarity ratio $\rho_{r}$, namely the fraction of filaments in the bundle oriented in opposite direction. Each modelled bundle comprises on average 73 filaments, with a uniform length distribution of $86-282 \mathrm{~nm}$ and a uniform distance distribution of 10-37 nm between neighboring filaments.

In total we created three ground truth datasets with three different SNRs, that are $0.01,0.001$, and 0.0001 . Each dataset consists of ten tomograms of different bundles, that are oriented in $10^{\circ}$ steps from $\varphi=0^{\circ}$ (filaments $\|$ to the tilt-axis) to $\varphi=90^{\circ}$ (filaments $\perp$ to the tilt-axis). 
Additionally, within each set we varied the polarity ratio $\rho_{r}$ in triplets, meaning that the first four tomograms $\left(\varphi=0^{\circ}, 10^{\circ}, 20^{\circ}, 30^{\circ}\right)$ were modelled with $\rho_{r}=0.1,0.3,0.5,0.1$, and so forth, except

120 the last tomogram $\left(\varphi=90^{\circ}\right)$ was constructed with a polarity ratio of 0.5 .

121 A top view on one of the modeled bundles $\left(\varphi=30^{\circ}, \rho_{r}=0.1\right)$ is shown in Fig. $1 \mathrm{~b}$ (without noise, only for visualization purposes). A second example is depicted in Fig. 1c. This bundle is oriented $70^{\circ}$ with respect to the tilt-axis and the polarity ratio is 0.3 . Its side view is displayed in Fig. 1d, with the viewing axis oriented parallel to the filaments. A bandpass filtered slice of a filament, that was boxed out of one of the ground truth tomograms $\left(\varphi=0^{\circ}, \mathrm{SNR}=0.001\right)$, is shown and can be compared to a filament parallel to the tilt-axis as well, but modelled with a

127 SNR of 0.0001 (Fig. 1e and f, respectively).

We applied the APT workflow on each of the three ground truth datasets independently. Since the segmentation of the filaments is given by the ground truth data, we first established the segment coordinates (Step I), with an equidistant spacing of $11 \mathrm{~nm}$, corresponding to $\sim 1000$ segments per modelled bundle. Next, the subtomograms were extracted using a box size of $50 \times 50 \times 50 \mathrm{~nm}^{3}$ (Step II) and subsequently projected using a projection thickness of $11 \mathrm{~nm}$ (Step III). In the next step segments were prealigned (Step IV), followed by the 2D classification module (Step V). In Supplemental Fig. 4 the results of prealignment and 2D classification are shown for the modelled dataset with $\mathrm{SNR}=0.0001$.

Following this, we executed $3 \mathrm{D}$ reconstruction (Step $\mathrm{VI}$ ) of the three actin filament averages

137 from a similar number of particles ( 10'000 segments per average), distributed approximately uniform over the modelled bundles. As expected, the resolutions of the filament structures, 139 drop with decreasing SNR (Supplemental Fig. 5).

140 Subsequently, we performed polarity determination (Step VII) of all modelled bundles. 141 All $\psi^{-1}$ histograms (see Method Details) showed two distinct peaks (Fig. 1g), separated by $142180^{\circ}$, indicating the opposite plus-end orientations of the segments, and the height of the peaks 143 reproduce the respective polarity ratios. All restored bundles from all three SNR conditions 144 showed satisfactory agreement with the ground truth bundles (Fig. 1h-i). Furthermore, we 
145 visualized the $\xi\left(F_{i}^{j}\right)$ vectors (see Method Details) of all the recovered bundles (Fig. 1j). The 146 position of incorrect assigned segments is randomly distributed and their number increases 147 with decreasing SNR.

148 The results of the confidence analysis (Step VIII) are plotted in Fig. 1k. The ccs values (see

149 Method Details) of bundles with $\mathrm{SNR}=0.001$ dropped slightly in comparison to bundles with $150 \mathrm{SNR}=0.01$, but show no trend to lower ccs values with increasing $\varphi$ angle. We conclude, that 151 the missing wedge induced anisotropic deterioration does not negatively influence the 152 precision of APT for low SNR values, as found in cellular cryo-tomograms (Forster et al., 2008; 153 Pei et al., 2016). However, for the markedly challenging SNR $=0.0001$ the ccs values decrease 154 with increasing $\varphi$ angle.

155 Finally, we evaluated the total segment error (Fig. 1k), namely the fraction of segments with 156 incorrect orientation determination compared to the ground truth. For low SNR conditions the 157 total segment error is $\lesssim 10 \%$ for all modelled bundles, and increases to $\sim 20 \%$ for bundles 158 modelled with SNR $=0.0001$. However, if we evaluate the total filament error, that is the fraction 159 of filaments with incorrect polarity determination compared to the ground truth, we find that the 160 polarity of solely $5 \%$ of the filaments (34 out of 712 filaments) were determined incorrectly for 161 the dataset modelled with $\mathrm{SNR}=0.0001$. This shows that APT is capable to reliably correct 162 noise induced polarity errors on the filament level. 
165 Reconstruction of actin filaments at FAs. Here we applied APT on the actin cytoskeleton

166 inside FAs, using a correlative fluorescence microscopy and cryo-ET approach (Patla et al., 167 2010; Sartori et al., 2007) (see Method Details). Mouse embryonic fibroblasts (MEFs), 168 expressing vinculin-venus as a marker for FAs (Grashoff et al., 2010; Ringer et al., 2017), were cultured on electron microscopy grids with silicon-oxide support and imaged by fluorescence microscopy (Fig. 2a). Conspicuous FAs were identified and found again under the electron

171 beam.

172 Due to the sheer size of FAs, tomograms cannot cover a complete adhesion site $(\sim 2-4 \mu \mathrm{m}$ in 173 length), and due to electron dose sensitivity of the sample, only a single tomogram can be acquired per FA. Therefore, we recorded cryo-tomograms at a spectrum of positions, namely from proximal regions, where a stress fibre enters the FA, to distal regions, where they are oriented towards the plasma membrane. Seven cryo-tomograms of FAs (Fig. 2b), covering

177 positions from proximal to distal, were selected in the first place, and the actin filaments were 178 manually segmented (Fig. 2c).

179 Following this, we applied the APT workflow on this dataset. Firstly, we extracted a total of 43'400 segments with an equidistant spacing of $11 \mathrm{~nm}$ (Step 1). The box size of the

181 subtomograms was set to $50 \times 50 \times 50 \mathrm{~nm}^{3}$ (Step II), and the projection thickness to $11 \mathrm{~nm}$ 182 (Step III). After prealignment and 2D classification (Step IV-V), we finally selected 20'585 segments (Supplemental Fig. 6a) for 3D reconstruction (Step VI).

184 The obtained in-situ actin filament structure was resolved to $18.4 \AA$, and allows to 185 unambiguously detect the position of its plus-end (Supplemental Fig. 6b-d). This shows that 186 the APT workflow is capable to produce sufficient resolution for subsequent mapping of the 187 filament's polarity distribution, although the dataset was relatively limited and the filaments 188 originate from a crowded and dense cellular environment, with multiple possible binding 189 partners and modulations of their helical symmetry.

190 In order to efficiently increase the size of the dataset, we used the previous seven manual 191 segmentations to train a convolutional neural network with EMAN2 (Chen et al., 2017), capable 
192 of detecting actin filaments (see Method Details). Using this approach, we additionally created

19331 segmentations of the actin cytoskeleton at FAs.

194 We then applied the APT workflow to this extended dataset. Here we extracted a total of 195247 '940 segments (with spacing of $11 \mathrm{~nm}$ ), using a box size of $36 \times 36 \times 36 \mathrm{~nm}^{3}$ and a projection thickness of $11 \mathrm{~nm}$ (Step I-III). After prealignment and 2D classification (Step IV-V), we selected 72'973 segments (Supplemental Fig. 7, Fig. 2d) for 3D reconstruction (Step VI). Subsequently, we performed a 3D classification with RELION (Supplemental Fig. 8, Supplemental Fig. 9). The highest resolved class average with $13.5 \AA$ is shown in Fig. $2 \mathrm{e}$, together with a docking of EMD-6179 (Galkin et al., 2015) in Fig. $2 f$.

Polarity distribution of actin bundles at FAs. Finally, we utilized APT to reconstruct actin networks in-situ, including the polarity of the filaments.

Therefore, we continued the APT workflow with the polarity determination module (Step VII), based on the in-situ actin filament reconstruction (Supplemental Fig. 6), previously obtained from seven manual segmentations of actin bundles at FAs. All $\psi^{-1}$ histograms showed two distinct peaks, separated by $180^{\circ}$. We extracted the $\xi\left(F_{i}^{j}\right)$ vectors of the resulting 2893 filaments, and used APT to calculate the confidence scores (Step VIII), plotted in Fig. 3a-c. The minimum reliable ccs was set to 0.6 (Fig. 3c), thereby leaving a total amount of 2146 actin filaments with determined polarity. The total length of the filaments is $\sim 149 \mu \mathrm{m}$, with an average length of $\sim 70 \mathrm{~nm}$, and a maximum length of $\sim 350 \mathrm{~nm}$ (Supplemental Fig. 10a,b).

In Fig. 3d the resulting $\xi\left(F_{i}^{j}\right)$ vectors of one of the bundles are displayed. Here we found that $62 \%$ of the segments are pointing with their plus-ends towards the cell tip (blue segments), and $38 \%$ in opposite direction (red segments). The actin polarity distribution of this actin bundle is visualized in Fig. 3e. The blue filaments are pointing with their plus-ends towards the cell tip, and the red filaments in opposite direction.

The two bundles shown in Fig. 3e-f were acquired at proximal regions of FAs, whereas the two bundles shown in Fig. 3g-h were acquired at distal regions. We applied APT's topology module 
(Step IX), and sorted the bundles with increasing topology score (Supplemental Fig. 10c) from left $(\tau=0.25)$ to right $(\tau=0.87)$, which reflects their transition from a tendentially mixed polarity distribution to a predominantly uniform polarity distribution. In Fig. $3 e, 65 \%$ of the filaments are directed towards the cell tip, whereas in Fig. 3h this fraction is increased to $96 \%$. The bundles in Fig. 3e and Fig. 3h are shown from the side in Fig. 3i and Fig. 3j. Furthermore, the long axis of the bundle in Fig. $3 e$ is shown in Fig. 3k. The data suggests that filaments, which are directed towards the cell tip, forming the core of the FA actin bundle, and the reversely directed filaments are organized around this core. The positive topology scores of bundles with mixed polarity distributions are a further indication for this architectural principle. If the reversed actin filaments would be diffused into the bundles, the topology score would approach negative values. In addition, we analysed the polarity distribution in layers parallel to the support, finding that reversed filaments are enriched on top and bottom of the mixed polarity bundles (Supplemental Fig. 10d).

232 The number of filaments within neighbourhood spheres $\mathcal{K}_{\epsilon}\left(x_{k}\right)$, which were evaluated during 233 topology analysis (Step IX) with a radius of $\epsilon=40 \mathrm{~nm}$, has its maximum at 6 neighbours 234 (Supplemental Fig. 10e). This indicates that actin filaments in FA bundles resemble a hexagonal packing with a mean filament distance of $28 \mathrm{~nm}$ (Supplemental Fig. 10f). Finally, we completed the APT workflow (Step VII-IX) for the extended dataset with previously obtained 31 automatic segmentations (Fig. 2d-f, Supplemental Fig. 7-9). Seven tomograms were excluded, because they contained ill-defined or multiple bundles with similar orientation, so that the $\psi^{-1}$ histograms did not exhibit two clearly defined peaks. As before, we set the 240 minimal reliable ccs to 0.6 , thereby leaving a total amount of 6152 actin filaments with 241 determined polarity. The total length of the analyzed filaments was increased to $\sim 388 \mu \mathrm{m}$. In Fig. 4 we plotted the topology score $\tau$ of these 24 bundles versus their normalized position along a FA (see Method Details). Strikingly, a pronounced uniform polarity distribution (high $\tau$ values) can be found in distal regions of FAs. For most of the analyzed bundles (Fig. 4, green dots) the plot suggests a correlation between topology score and normalized FA position. In other words, within a FA, from proximal to distal regions, the polarity distribution of the actin 
247 filaments transforms smoothly from mixed to uniform actin polarity organization. Interestingly,

248 this correlation does not hold true, when the FA is not positioned towards a pronounced cell

249 protrusion (Fig. 4, orange dots).

\section{Discussion}

252 Resolving the polarity of actin filaments inside cells, would provide detailed insights into 253 cytoskeletal processes and their functional organization. Therefore, we implemented APT, a 254 set of MATLAB scripts and RELION procedures that enables the reconstruction of actin 255 filaments and the assessment of their polarity. This general approach can be applied to any 256 cryo-tomograms, in which actin filaments are observed. Moreover, the usage of APT to reconstruct in-situ actin filament structures, opens new avenues to explore complex actin networks and even structural interactions of actin and actin binding proteins.

259 The basic concept of APT is to dissect an actin filament into multiple segments, and 260 subsequently measure their polarity with single particle techniques. Thereby we create a set of statistically independent estimations of the filament's polarity. Based on majority, the final polarity of the filament is determined. We validated this approach and found that the total filament error is below $5 \%$, even for very challenging and low SNR $=0.0001$. A previous approach to decipher actin polarity from tomographic data necessitated chemical extraction and negative staining of the cytoskeleton (Narita et al., 2012), in order to increase

266 the SNR of the tomograms. However, the challenge of resolving actin filament structure and 267 polarity in-situ requires a tomographic data set from intact cells (Beck and Baumeister, 2016; 268 Lucic et al., 2005), which involves vitrification of the sample, and therefore the method needs 269 to successfully handle low SNR (Forster et al., 2008; Pei et al., 2016).

270 As an example, we applied APT on actin bundles found at FAs. These multi-layered 271 assemblies, around 200-350 nm in thickness, connect integrin receptors, which anchor the 272 plasma membrane to the extracellular matrix, via force transduction and actin regulation layers, 273 with the actin cytoskeleton (Kanchanawong et al., 2010; Patla et al., 2010). 
Actin bundles at FAs (Fig. 3e-k) resemble a hexagonal packing of the filaments with an average distance of $\sim 28 \mathrm{~nm}$ (Supplemental Fig. 10e,f). The majority of the filaments is directed towards the cell tip (Fig. 3e-h), and form the core of the bundles, whereas filaments with reversed direction organize around this core (Fig. 3i-k, Supplemental Fig. 10d). Ultimately, the polarity distribution depends on the position of the bundle along the FA (Fig. 4).

279 The more distal the position, the more uniform the polarity distribution, while mixed polarity can 280 be observed mainly in the proximal regions (Fig. 5). Stress fibers are contractile actin 281 assemblies (Malik-Garbi et al., 2019), enriched with actomyosin interactions, which favor a mixed polarity distribution of actin filaments. Proximal regions of FAs anchor to stress fibers (Burridge and Guilluy, 2016), therefore exhibiting a mixed polarity actin bundle. However, at distal regions of FAs, more vectoral or even protruding forces may play a role that are resembled in a more uniform polarity distribution.

286 Technical developments in sample preparation allowed cryo-ET to provide fundamental 287 insights into cellular assemblies and processes in-situ (Mahamid et al., 2016; Marko et al., 288 2007; Schaffer et al., 2019). However, it also requires the development of novel tools to analyse the data (Chen et al., 2017; Himes and Zhang, 2018; Martinez-Sanchez et al., 2020; Song et al., 2019). APT complements these tools and allows to analyse actin filaments in physiological relevant processes, which would provide a better understanding of actin networks, such as the actin cortex (Chugh et al., 2017), stereocilia organization (Metlagel et al., 2019) or remodelling of actin during phagocytosis (Gerisch, 2011).

\section{Method Details}

296 Implementation and code availability. All functionality of APT was implemented as MATLAB 297 (MathWorks, Natick, USA) scripts and functions. In order to interface with RELION (Scheres, 2012) (tested with version 3.0.4), respective functions to write and read starfiles were 299 programmed.

300 The software is available at https://www.placeholder.com. It is equipped with a tutorial that 301 explains APT step-by-step based on modelled tomograms of actin bundles. 
303 Step I: Coordinates. The input data is a set of $N_{T}$ cryo-electron tomograms $T_{i}\left(i=1, \cdots, N_{T}\right)$ and for each $T_{i}$ there is an associated segmentation $S_{i}$ of the actin filaments. Each $S_{i}$ define a set of filaments $F_{i}^{j}\left(j=1, \cdots, N_{i}\right)$, with $N_{i}$ being the number of detected filaments in $T_{i}$. Firstly, APT utilizes the $S_{i}$ to construct for each $F_{i}^{j}$ a set of $3 \mathrm{D}$ coordinates $\left\{x_{1}, x_{2}, \cdots, x_{N_{i}^{j}}\right\}$, that are evenly spaced along $F_{i}^{j}$, and will be the sampling points for polarity determination. Here $N_{i}^{j}$ indicates the number of sampling points per filament, which we term segments.

Step II: Subtomograms. Next, APT pools all these $N_{C}=\sum N_{i}^{j}$ segments $x_{k}\left(k=1, \cdots, N_{C}\right)$,

stores the index relation $k \leftrightarrow F_{i}^{j}$ for polarity determination at a later stage, and extracts $N_{C}$ subtomograms centred around $x_{k}$ from a ctf-corrected version of the dataset $T_{i}$.

Step III: Projections. Subsequently, APT masks and then projects the subtomograms in z-direction. The applied mask diminishes the influence of neighbouring filaments in z-direction. We term the height of the mask as projection thickness parameter.

Step IV: Prealignment. This is the first of three steps performed in RELION. First, a starfile was created only passing the name of each projected subtomogram ( $r$ ln ImageName) and its originating tomogram ( $r$ InMicrographName) to RELION. Next, the projected subtomograms were normalized with relion_preprocess. Please note that the index relation $k \leftrightarrow F_{i}^{j}$ was kept invisible for RELION in all the following steps (for example no metadata label

323 rlnHelicalTubeID was provided (He and Scheres, 2017)). Furthermore, CTF correction in

324 RELION jobs was switched off, because the tomograms were CTF corrected by phase-flipping 325 prior to subtomogram extraction.

326 For prealignment of the segments a 2D classification with the prepared starfile as input was 327 performed. The purpose of this step is to align the (central) actin filament density parallel with 
328 the $x$-axis, which is the RELION convention for helical reconstruction (He and Scheres, 2017).

329 Therefore, we created a set of projection images (the template library) from an actin filament

330 structure, which was oriented along the x-axis, rotated in $3^{\circ}$ increments and subsequently

331 projected. The template library was used as initial references for prealignment (Supplemental

332 Fig. 4a).

333 Since the resolution of the actin filament averages was determined by Fourier shell correlation 334 between the averages and EMD-6179 (Galkin et al., 2015) as an external reference 335 (Supplemental Fig. 5, Supplemental Fig. 6d, and Supplemental Fig. 9), we were reluctant to use the same structure for the construction of the template library. For that purpose, we used EMD-10737.

Step V: 2D Classification. Subsequently we performed a second 2D classification in RELION, 340 passing the psi angles and translations found in the previous step as priors

341 (rlnAnglepsiprior, rlnoriginXPrior, rlnoriginyprior). In contrast to the 342 prealignment step, it is crucial that this $2 \mathrm{D}$ classification is unsupervised, in order to extract the 343 structural heterogeneity in the data unbiasedly (Supplemental Fig. 4c-f).

344 The psi search was conducted local around the psi prior. Therefore, it was possible to apply a second mask (the first mask was applied during the projection step in z-direction), which allowed to further diminish the influence of neighbouring filaments in y-direction.

347 This second 2D classification is capable of producing high quality class averages of 348 filamentous actin from inside intact cells (Supplemental Fig. 6a, Supplemental Fig. 7), and 349 allows to sort out false positive actin detections and low-quality segments (Bharat and Scheres, 2016). Based on this classification we selected the segments for subsequent 3D 351 reconstruction.

353 Step VI: 3D Reconstruction. For actin filament averaging a 3D refine job in RELION was 354 performed with helical reconstruction switched on (He and Scheres, 2017). As helical rise 27.6 $355 \AA$ A and as helical twist $166.7^{\circ}$ was used (Galkin et al., 2015). In the input starfile, the refined psi 
angle and translations found in the previous step were passed as priors plus for each segment a random rot angle as prior was added and the tilt prior was set to $90^{\circ}$ (He and Scheres, 2017) (rlnAngleRotPrior, rlnAngleTiltPrior). As initial template the same structure as for construction of the template library was used, low-pass filtered to $30 \AA$ (Supplemental Fig. 6d). transformation $\Omega_{k}$, that describes how filament $F_{i}^{j}$ has to be transformed at sampling point $x_{k}$ in order to align with the filament average (Supplemental Fig. 3a).

Step VII: Polarity. To resolve actin polarity, it is crucial that the filament average reaches a resolution better than $20 \AA$. Once this has been achieved, APT calculates the inverse transformation $\Omega_{k}^{-1}$, that describes how filament $F_{i}^{j}$ is oriented at sampling point $x_{k}$ with respect inverted psi angle $\psi_{k}^{-1}$, which indicates the position of the plus-end of $F_{i}^{j}$ relative to the $\mathrm{y}$-axis of $T_{i}$.

Next, APT uses the index relation $k \leftrightarrow F_{i}^{j}$ to extract all inverted psi angles $\psi_{k}^{-1}\left(T_{i}\right)$, that are originating from the same $T_{i}$. If $T_{i}$ contains a filament bundle with mixed polarity, then the histogram of $\psi_{k}^{-1}\left(T_{i}\right)$ shows two distinct peaks, that are separated by $180^{\circ}$. The height of each peak indicates how many segments were aligned with their plus-ends in the respective 374 direction, and allows an estimation of the polarity ratio within the bundle, while the peak width 375 decreases with increasing parallelism of the filaments. Obviously, if a bundle exhibits a uniform 376 polarity distribution, then the histogram of $\psi_{k}^{-1}\left(T_{i}\right)$ shows a single peak.

377 Subsequently, APT assigns to each segment a direction label. Per default, segments included 378 in the right peak are labelled with 0 , segments included in the left peak are labelled with 1. 379 Therefore, each filament can be represented as a vector $\xi\left(F_{i}^{j}\right)$ of labelled segments.

380 The centrepiece of APT is that the elements of $\xi\left(F_{i}^{j}\right)$ are computed statistically independent. 381 Segments, whose directionality was detected incorrectly, do not prohibit the correct polarity 382 determination of a filament as a whole, as long as the majority of segments building that 
383

384

385

386

387

388

389

390

391

392

393

394

395

396

397

398

399

400

401

402

403

filament were determined accurately. Consequently, the final polarity label $P\left(F_{i}^{j}\right)$ of filament $F_{i}^{j}$ is defined as follows:

$$
P\left(F_{i}^{j}\right)= \begin{cases}0 & \text { if mean }\left(\xi\left(F_{i}^{j}\right)\right)<0.5 \\ 0.5 & \text { if mean }\left(\xi\left(F_{i}^{j}\right)\right)=0.5 \\ 1 & \text { if mean }\left(\xi\left(F_{i}^{j}\right)\right)>0.5\end{cases}
$$

If $P\left(F_{i}^{j}\right)=0.5$ the polarity of that filament is maximally uncertain.

Step VIII: Confidence. We suggest that the closer mean $\left(\xi\left(F_{i}^{j}\right)\right)$ is to 0 or to 1 , respectively, the higher the probability, that $P\left(F_{i}^{j}\right)$ was determined correctly. We use this relation to define a confidence score for each filament, termed majority confidence score (mcs), as follows:

$$
\operatorname{mcs}\left(F_{i}^{j}\right)=\max \left(\operatorname{mean}\left(\xi\left(F_{i}^{j}\right)\right), \operatorname{mean}\left(\sim \xi\left(F_{i}^{j}\right)\right)\right),
$$

with $\sim \xi\left(F_{i}^{j}\right)$ the logical complement of $\xi\left(F_{i}^{j}\right)$. The values of mcs range between 0.5 (maximum uncertainty) to 1 (minimal uncertainty), and are the fraction of segments per filament that are pointing in the majority direction. The mcs measures the consistency of the segment's directionality determination along a filament.

Additionally, we define a second confidence score, termed sensitivity confidence score (scs). Here the reconstruction of the filament average is repeated with a reversed direction of the template. Subsequently, APT initializes for each $F_{i}^{j}$ a vector $\lambda\left(F_{i}^{j}\right)$, having the same number of elements than $F_{i}^{j}$ segments. Those segments that reverse their plus-end orientation as well, are orientational sensitive and APT sets the respective elements of $\lambda\left(F_{i}^{j}\right)$ to 1 . On the other hand, if a segment shows no logical behaviour upon change in template direction, the respective element of $\lambda\left(F_{i}^{j}\right)$ is set to 0 . Then we define scs as follows:

$$
\operatorname{scs}\left(F_{i}^{j}\right)=\operatorname{mean}\left(\lambda\left(F_{i}^{j}\right)\right)
$$


404 The values of scs range between 0 (no segment of $F_{i}^{j}$ is orientational sensitive) and $1\left(F_{i}^{j}\right.$ is

405

406

407

408

409

410

411

412

413

414

415

416

417

418

419

420

422

423

424

425

426

427

428 Ground truth data generator. In the first step of modelling a cryo-tomogram of an actin 429 built exclusively from orientational sensitive segments). Comparable to the mcs, the scs is an alternative to measure the self-consistency of the segment's directionality determination.

Finally, we define the combined confidence score (ccs) of filament $F_{i}^{j}$ as follows:

$$
\operatorname{ccs}\left(F_{i}^{j}\right)=\operatorname{mcs}\left(F_{i}^{j}\right) \cdot \operatorname{scs}\left(F_{i}^{j}\right)
$$

Step IX: Topology. In this module APT performs a 3D neighbourhood analysis of the actin filaments in order to characterize their polarity distribution in the observed bundles. Therefore, centred around each segment $x_{k}$, APT constructs a sphere $\mathcal{K}_{\epsilon}\left(x_{k}\right)$ with radius $\epsilon$, defining the neighbourhood of $x_{k}$. Then, APT extracts number, distance and polarity of all filaments passing through $\mathcal{K}_{\epsilon}\left(x_{k}\right)$. For instance, let the polarity of the segment $x_{k}$ be $P\left(F_{i}^{j}\right)=0$, and imagine $F_{i}^{j}$ is surrounded by four filaments within $\mathcal{K}_{\epsilon}\left(x_{k}\right)$, of which one displays same polarity and three reversed polarity (Supplemental Fig. 3b). Then, the position of the probed filament is located in a neighbourhood that exhibits $75 \%$ mixed polarity and $25 \%$ uniform polarity, respectively. This calculation is performed by APT for all $\mathcal{K}_{\epsilon}\left(x_{k}\right)$, and subsequently it sums the degrees of uniform polarity $P_{\|}$and mixed polarity $P_{\nVdash}$ of all neighbourhoods in a given actin bundle. Finally, the topology score $\tau$ of an actin bundle is defined as follows:

$$
\tau=\frac{P_{\|}-P_{\sharp}}{P_{\|}+P_{\sharp}} .
$$

The topology score $\tau$ ranges from -1 to +1 . The more the filaments are organized in a uniform polarity configuration, the value of $\tau$ will be closer to +1 . If the bundle shows a phase separated topology, so that filaments with opposite polarities adjoin at phase boundaries mainly, $\tau$ will be reduced accordingly. However, the more the bundle favours close proximity between filaments with opposite polarities, the more $\tau$ will approach -1 .

bundle, filament $x$-z-coordinates were calculated based on random close packing (Desmond 
and Weeks, 2009), with a uniform distance distribution in the range between 10-37 nm. As a consequence of the modelled x-z-dimension of the bundles of $282 \times 150 \mathrm{~nm}$, on average 73 filaments could be packed in each bundle. Next, the position and length of each filament in ydirection was randomly chosen from a uniform distribution between 86-282 $\mathrm{nm}$. According to these coordinates, actin filaments based on EMD-6179 (Galkin et al., 2015), were pasted in an empty volume of $353 \times 353 \times 353 \mathrm{~nm}^{3}$, however, a random fraction of $\rho_{r}$ filaments was pasted in the volume after $180^{\circ}$ rotation around the $\mathrm{x}$-axis (Fig. $1 \mathrm{~b}$-d, red coloured 437 filaments).

After that the modelled bundle was rotated $\varphi$ degrees around the z-axis, then Gaussian noise was added to adjust the targeted SNR, and finally the missing wedge of a tilt-series with tiltrange from $-60^{\circ}$ to $+60^{\circ}$ was applied in Fourier space.

441 Cell culture. MEFs stably expressing vinculin-venus were cultured in Dulbecco's Modified 442 Eagle's Medium (Sigma-Aldrich, D5671), supplemented with 10\% (v/v) fetal bovine serum 443 (Sigma-Aldrich, G7524), 2 mM L-glutamine (Sigma-Aldrich, G7513) and $100 \mu \mathrm{g} / \mathrm{ml}$ penicillin444 streptomycin (Sigma-Aldrich, P0781), at $37^{\circ} \mathrm{C}$ and $5 \% \mathrm{CO}_{2}$. The MEFs were applied onto 445 glow-discharged EM grids with a silicon-oxide support film (R1/4, Au mesh; Quantifoil, Jena, Germany). After incubation, the cells were fixed in a $4 \%$ paraformaldehyde solution (SigmaAldrich, 16005), and washed 3 times with 1x PBS (Fisher Scientific, BP399-1).

Fluorescence microscopy. The EM grids were transferred to a $35 \times 14 \mathrm{~mm}$ glass-bottom cell 450 culture dish (MatTek, P35G-0-14-C) for fluorescence microscopy analysis of the FAs. Adherent 451 MEFs were imaged using an automated inverted microscope (DMI4000 B, Leica 452 Microsystems, Wetzlar, Germany) equipped with a fluorescence lamp and a monochromatic 453 digital camera (DFC365 FX, Leica Microsystems). Overview images of the EM grids were 454 acquired using a 10x dry objective (HCX PL Fluotar 10x/0.30, Leica Microsystems). All images 455 of individual cells (Fig. 2a) were acquired in phase contrast and fluorescence mode using a 456 63x oil objective (HCX PL APO 63x/1.40-0.6, Leica Microsystems). 
458 Cryo-electron tomography. A drop of $4 \mu \mathrm{l} \mathrm{BSA-coated} 10 \mathrm{~nm}$ fiducial gold markers (Aurion, 459 Wageningen, Netherlands) was applied on the EM grids before plunging them into liquid 460 nitrogen cooled ethane.

461 A Titan Krios transmission electron microscope (Thermo Fisher Scientific, Waltham, USA) 462 equipped with a Quantum energy filter and a K2-Summit direct electron detector (Gatan, 463 Pleasanton, USA) was used for cryo-EM data acquisition. The microscope was operated at $464300 \mathrm{keV}$ in zero-loss mode with the energy filter slit width set to $20 \mathrm{eV}$.

465 The position of the cells and FAs were identified by overlaying the fluorescence signal to low 466 magnification EM images of the grids. At tomography positions image stacks were recorded at 467 each tilt angle in super-resolution mode with an electron flux of $\sim 8$ electrons per pixel per 468 second using SerialEM (Mastronarde, 2005). All tomograms were acquired at a magnification of $42^{\prime} 000 \mathrm{x}$, and a dose-fractionated frame rate of 6 frames per $1.2 \mathrm{~s}$. The tilt-series covered

470 an angular range of $-60^{\circ}$ to $+60^{\circ}$, and were recorded with tilt increments of $2^{\circ}$ and a defocus 471 of $-4 \mu \mathrm{m}$. The accumulated electron dose did not exceed $\sim 75 \mathrm{e}^{-/ \AA^{2}}$.

472 All image stacks were down-sampled and motion corrected using MotionCorr (Li et al., 2013),

473 resulting in a final pixel size of $3.44 \AA$. Next, CTF correction of the tilt-series was applied 474 (Eibauer et al., 2012). Overview tomograms (Fig. 2b) for actin filament segmentation (Fig. 2c) 475 and subtomograms of actin segments were reconstructed by weighed back-projection using 476 the TOM Toolbox (Nickell et al., 2005).

478 Normalized bundle position of FA. The tomographic position within a FA was determined by 479 overlaying the light microscopy images of the individual cells with cryo-EM images.

480 First, phase contrast and fluorescence images were overlaid, hereby enabling the identification 481 of the cell membrane and enclosing grid holes. Second, low magnification EM images (4'800x) of the FA tomography position were overlaid such, that the cell membrane and grid holes 483 coincided with the light microscopy images. Third, the zero-degree tilt-series image (42'000x) 484 was aligned with the low magnification EM image such, that the cell membrane shape 
matched. By increasing the opacity of the EM images, we displayed the FA fluorescence signal on the tomography position.

487 We defined the center of the tomogram as the tomography position $F A_{T}$. In order to determine 488 the relative position of $F A_{T}$ within the entire $F A$, we defined the proximal end (closest to cell body) and distal end (closest to cell periphery) fluorescence microscopy signal pixels of the FA as $F A_{P}$ and $F A_{D}$, respectively. The distance $d$ between $F A_{T}$ and $F A_{D}$ and the length $l$ of the $F A$

491 (distance between $F A_{P}$ and $F A_{D}$ ) was measured (the pixel size of the fluorescence microscopy 492 images was $91 \mathrm{~nm}$ ). Finally, the normalized bundle position of the FA (plotted on the y-axis in Fig. 4) was calculated as $1-d / l$.

Automatic segmentation. We developed a script in MATLAB to automatize the convolutional neural network (CNN) segmentation with EMAN2 (Chen et al., 2019; Chen et al., 2017). The method requires three image stacks with fixed $x-y$-dimension of $64 \times 64$ pixels as input 498 files. Firstly, the positive training stack contains 2D images of densities targeted for 499 segmentation. These images are slices, boxed out of cryo-tomograms. In our case they contain 500 actin filament densities. Secondly, an image stack that provides for each image in the positive 501 training stack an associated segmentation mask. Thirdly, the negative training stack, which contains 2D images of densities not targeted for segmentation, for example gold particles used as fiducial markers, backprojection rays, tomogram edges, plasma membrane, and regions without actin filaments in general.

505 As a starting point we created seven manual segmentations of actin filaments in cryo506 tomograms of FA actin bundles (Fig. 2c) with AMIRA (Thermo Fisher Scientific, Waltham, 507 USA). The segmentations were skeletonized, then the filaments were dissected in segments, 508 and around each 3D coordinate of a segment one positive training image was extracted from 509 the tomogram at respective z-position. For each manual segmentation one positive training 510 stack was created, limited to 3'000 images.

511 In order to create the associated segmentation masks, we extended the skeletonized filaments 512 to the diameter of an actin filament. Subsequently, the segmentation masks were extracted 
513 from the extended segmentation at the same coordinates as the positive training images. For

514 each manual segmentation one stack with segmentation masks was created, with the same

515 dimension as the associated positive training stack.

516 Negative training images were extracted at random positions from the tomograms, but the

517 volume of the actin bundle was excluded. For each manual segmentation one negative training

518 stack was created, limited to 3'000 images as well.

519 Based on these image stacks, we trained one CNN for each manually segmented tomogram, 520 using default EMAN2 data augmentation, network and training parameters. In the next step,

521 the resulting seven CNNs were applied to each of the 31 tomograms in the extended dataset,

522 thereby creating a total amount of 217 segmentations. Finally, the segmentations belonging to

523 the same tomogram were averaged and post-processed in UCSF Chimera (Pettersen et al.,

524 2004) with the hide dust command.

525

526 Visualization. All isosurface visualizations of actin filament structures and actin bundles were

527 rendered with UCSF Chimera or AMIRA.

528 For the visualizations of actin bundles in Fig. 3e-k we used EMD-6179 (Galkin et al., 2015) to 529 represent the filaments. Therefore, we used a b-spline registration algorithm to bend the 530 filaments in order to match their 3D shape defined by the segment coordinates (Rueckert et 531 al., 1999).

532

533 Data availability. Actin filament averages will be deposited in the Electron Microscopy Data 534 Bank under accession codes EMD-abcd ${ }_{1}$ for the actin filament structure obtained from the 535 manual segmented dataset (Supplemental Fig. 6), and EMD-abcd 2 , EMD-abcd 3 , and 536 EMD-abcd $_{4}$ for the actin filament class averages (Supplemental Fig. 8) obtained from the extended dataset. 


\section{Acknowledgements}

541 This work was funded by grants from the Swiss National Science Foundation (SNSF 542 31003A_179418), ERC-Syg and the Mäxi Foundation to O.M. We thank Carsten Grashoff for 543 the vinculin labelled MEF cells. We thank the Center for Microscopy and Image Analysis at the 544 University of Zurich.

545

\section{Author contributions}

547 B. M. prepared samples and recorded data. M. E. developed and implemented the method. 548 B. M., S. S., W. C., and M. T. wrote and tested code. B. M. and M. E. analyzed data. O. M. 549 and M. E. conceived the research and wrote the manuscript with input from all authors.

550

\section{Competing interests}

552 The authors declare no competing interests.

553

554 


\section{References}

Beck, M., and Baumeister, W. (2016). Cryo-Electron Tomography: Can it Reveal the Molecular Sociology of Cells in Atomic Detail? Trends Cell Biol 26, 825-837.

Bharat, T.A.M., and Scheres, S.H.W. (2016). Resolving macromolecular structures from electron cryo-tomography data using subtomogram averaging in RELION. Nat Protoc 11, 9-20.

Burridge, K., and Guilluy, C. (2016). Focal adhesions, stress fibers and mechanical tension. Exp Cell Res 343, 14-20.

Chen, M., Bell, J.M., Shi, X., Sun, S.Y., Wang, Z., and Ludtke, S.J. (2019). A complete data processing workflow for cryo-ET and subtomogram averaging. Nat Methods 16, 1161-1168.

Chen, M., Dai, W., Sun, S.Y., Jonasch, D., He, C.Y., Schmid, M.F., Chiu, W., and Ludtke, S.J. (2017). Convolutional neural networks for automated annotation of cellular cryoelectron tomograms. Nat Methods 14, 983-985.

Chugh, P., Clark, A.G., Smith, M.B., Cassani, D.A.D., Dierkes, K., Ragab, A., Roux, P.P., Charras, G., Salbreux, G., and Paluch, E.K. (2017). Actin cortex architecture regulates cell surface tension. Nat Cell Biol 19, 689-697.

Desmond, K.W., and Weeks, E.R. (2009). Random close packing of disks and spheres in confined geometries. Phys Rev E 80 .

Egelman, E.H., Francis, N., and DeRosier, D.J. (1982). F-actin is a helix with a random variable twist. Nature 298, 131-135.

Eibauer, M., Hoffmann, C., Plitzko, J.M., Baumeister, W., Nickell, S., and Engelhardt, H. (2012). Unraveling the structure of membrane proteins in situ by transfer function corrected cryo-electron tomography. Journal of Structural Biology 180, 488-496.

Elad, N., Volberg, T., Patla, I., Hirschfeld-Warneken, V., Grashoff, C., Spatz, J.P., Fassler, R., Geiger, B., and Medalia, O. (2013). The role of integrin-linked kinase in the molecular architecture of focal adhesions. J Cell Sci 126, 4099-4107.

Forster, F., Medalia, O., Zauberman, N., Baumeister, W., and Fass, D. (2005). Retrovirus envelope protein complex structure in situ studied by cryo-electron tomography. Proc Natl Acad Sci U S A 102, 4729-4734.

Forster, F., Pruggnaller, S., Seybert, A., and Frangakis, A.S. (2008). Classification of cryoelectron sub-tomograms using constrained correlation. J Struct Biol 161, 276-286.

Galkin, V.E., Orlova, A., Vos, M.R., Schroder, G.F., and Egelman, E.H. (2015). Near-atomic resolution for one state of F-actin. Structure 23, 173-182.

Geiger, B., Spatz, J.P., and Bershadsky, A.D. (2009). Environmental sensing through focal adhesions. Nat Rev Mol Cell Biol 10, 21-33.

Gerisch, G. (2011). Actin switches in phagocytosis. Commun Integr Biol 4, 344-345.

Grashoff, C., Hoffman, B.D., Brenner, M.D., Zhou, R., Parsons, M., Yang, M.T., McLean, M.A., Sligar, S.G., Chen, C.S., Ha, T., et al. (2010). Measuring mechanical tension across vinculin reveals regulation of focal adhesion dynamics. Nature 466, 263-266.

He, S., and Scheres, S.H.W. (2017). Helical reconstruction in RELION. J Struct Biol 198, 163176.

Hervas-Raluy, S., Garcia-Aznar, J.M., and Gomez-Benito, M.J. (2019). Modelling actin polymerization: the effect on confined cell migration. Biomech Model Mechanobiol 18, 1177-1187.

Himes, B.A., and Zhang, P. (2018). emClarity: software for high-resolution cryo-electron tomography and subtomogram averaging. Nat Methods 15, 955-961. 
602

603

604

605

606

607

608

609

610

611

612

613

614

615

616

617

618

619

620

621

622

623

624

625

626

627

628

629

630

631

632

633

634

635

636

637

638

639

640

641

642

643

644

645

646

647

648

Holmes, K.C., Popp, D., Gebhard, W., and Kabsch, W. (1990). Atomic model of the actin filament. Nature 347, 44-49.

Jasnin, M., Beck, F., Ecke, M., Fukuda, Y., Martinez-Sanchez, A., Baumeister, W., and Gerisch, G. (2019). The Architecture of Traveling Actin Waves Revealed by Cryo-Electron Tomography. Structure 27, 1211-1223 e1215.

Kanchanawong, P., Shtengel, G., Pasapera, A.M., Ramko, E.B., Davidson, M.W., Hess, H.F., and Waterman, C.M. (2010). Nanoscale architecture of integrin-based cell adhesions. Nature 468, 580-584.

Legate, K.R., Takahashi, S., Bonakdar, N., Fabry, B., Boettiger, D., Zent, R., and Fassler, R. (2011). Integrin adhesion and force coupling are independently regulated by localized PtdIns(4,5)2 synthesis. EMBO J 30, 4539-4553.

Li, X.M., Mooney, P., Zheng, S., Booth, C.R., Braunfeld, M.B., Gubbens, S., Agard, D.A., and Cheng, Y.F. (2013). Electron counting and beam-induced motion correction enable near-atomic-resolution single-particle cryo-EM. Nature Methods 10, 584-+.

Lucic, V., Forster, F., and Baumeister, W. (2005). Structural studies by electron tomography: from cells to molecules. Annu Rev Biochem 74, 833-865.

Mahamid, J., Pfeffer, S., Schaffer, M., Villa, E., Danev, R., Cuellar, L.K., Forster, F., Hyman, A.A., Plitzko, J.M., and Baumeister, W. (2016). Visualizing the molecular sociology at the HeLa cell nuclear periphery. Science 351, 969-972.

Malik-Garbi, M., Ierushalmi, N., Jansen, S., Abu-Shah, E., Goode, B.L., Mogilner, A., and Keren, K. (2019). Scaling behaviour in steady-state contracting actomyosin networks. Nat Phys 15, 509-516.

Marko, M., Hsieh, C., Schalek, R., Frank, J., and Mannella, C. (2007). Focused-ion-beam thinning of frozen-hydrated biological specimens for cryo-electron microscopy. Nat Methods 4, 215-217.

Martinez-Sanchez, A., Kochovski, Z., Laugks, U., Meyer Zum Alten Borgloh, J., Chakraborty, S., Pfeffer, S., Baumeister, W., and Lucic, V. (2020). Template-free detection and classification of membrane-bound complexes in cryo-electron tomograms. Nat Methods.

Mastronarde, D.N. (2005). Automated electron microscope tomography using robust prediction of specimen movements. Journal of Structural Biology 152, 36-51.

Medalia, O., Weber, I., Frangakis, A.S., Nicastro, D., Gerisch, G., and Baumeister, W. (2002). Macromolecular architecture in eukaryotic cells visualized by cryoelectron tomography. Science 298, 1209-1213.

Merino, F., Pospich, S., Funk, J., Wagner, T., Kullmer, F., Arndt, H.D., Bieling, P., and Raunser, S. (2018). Structural transitions of F-actin upon ATP hydrolysis at near-atomic resolution revealed by cryo-EM. Nat Struct Mol Biol 25, 528-537.

Metlagel, Z., Krey, J.F., Song, J., Swift, M.F., Tivol, W.J., Dumont, R.A., Thai, J., Chang, A., Seifikar, H., Volkmann, N., et al. (2019). Electron cryo-tomography of vestibular haircell stereocilia. J Struct Biol 206, 149-155.

Narita, A., Mueller, J., Urban, E., Vinzenz, M., Small, J.V., and Maeda, Y. (2012). Direct determination of actin polarity in the cell. J Mol Biol 419, 359-368.

Nickell, S., Forster, F., Linaroudis, A., Net, W.D., Beck, F., Hegerl, R., Baumeister, W., and Plitzko, J.M. (2005). TOM software toolbox: acquisition and analysis for electron tomography. J Struct Biol 149, 227-234.

Nogales, E. (2016). The development of cryo-EM into a mainstream structural biology technique. Nat Methods 13, 24-27. 
Patla, I., Volberg, T., Elad, N., Hirschfeld-Warneken, V., Grashoff, C., Fassler, R., Spatz, J.P., Geiger, B., and Medalia, O. (2010). Dissecting the molecular architecture of integrin adhesion sites by cryo-electron tomography. Nat Cell Biol 12, 909-915.

Pei, L., Xu, M., Frazier, Z., and Alber, F. (2016). Simulating cryo electron tomograms of crowded cell cytoplasm for assessment of automated particle picking. BMC Bioinformatics 17, 405.

Pettersen, E.F., Goddard, T.D., Huang, C.C., Couch, G.S., Greenblatt, D.M., Meng, E.C., and Ferrin, T.E. (2004). UCSF chimera - A visualization system for exploratory research and analysis. J Comput Chem 25, 1605-1612.

Pollard, T.D., and Borisy, G.G. (2003). Cellular motility driven by assembly and disassembly of actin filaments. Cell 112, 453-465.

Punjani, A., Rubinstein, J.L., Fleet, D.J., and Brubaker, M.A. (2017). cryoSPARC: algorithms for rapid unsupervised cryo-EM structure determination. Nat Methods 14, 290-296.

Ringer, P., Weissl, A., Cost, A.L., Freikamp, A., Sabass, B., Mehlich, A., Tramier, M., Rief, M., and Grashoff, C. (2017). Multiplexing molecular tension sensors reveals piconewton force gradient across talin-1. Nat Methods 14, 1090-1096.

Rueckert, D., Sonoda, L.I., Hayes, C., Hill, D.L.G., Leach, M.O., and Hawkes, D.J. (1999). Nonrigid registration using free-form deformations: Application to breast MR images. Ieee T Med Imaging 18, 712-721.

Sartori, A., Gatz, R., Beck, F., Rigort, A., Baumeister, W., and Plitzko, J.M. (2007). Correlative microscopy: bridging the gap between fluorescence light microscopy and cryoelectron tomography. J Struct Biol 160, 135-145.

Schaffer, M., Pfeffer, S., Mahamid, J., Kleindiek, S., Laugks, T., Albert, S., Engel, B.D., Rummel, A., Smith, A.J., Baumeister, W., et al. (2019). A cryo-FIB lift-out technique enables molecular-resolution cryo-ET within native Caenorhabditis elegans tissue. Nat Methods 16, 757-762.

Scheres, S.H. (2012). RELION: implementation of a Bayesian approach to cryo-EM structure determination. J Struct Biol 180, 519-530.

Schur, F.K., Obr, M., Hagen, W.J., Wan, W., Jakobi, A.J., Kirkpatrick, J.M., Sachse, C., Krausslich, H.G., and Briggs, J.A. (2016). An atomic model of HIV-1 capsid-SP1 reveals structures regulating assembly and maturation. Science 353, 506-508.

Shemesh, T., Geiger, B., Bershadsky, A.D., and Kozlov, M.M. (2005). Focal adhesions as mechanosensors: a physical mechanism. Proc Natl Acad Sci U S A 102, 12383-12388.

Song, K., Shang, Z., Fu, X., Lou, X., Grigorieff, N., and Nicastro, D. (2019). In situ structure determination at nanometer resolution using TYGRESS. Nat Methods.

Weber, M.S., Wojtynek, M., and Medalia, O. (2019). Cellular and Structural Studies of Eukaryotic Cells by Cryo-Electron Tomography. Cells 8.

$\mathrm{Xu}, \mathrm{K} .$, Babcock, H.P., and Zhuang, X. (2012). Dual-objective STORM reveals threedimensional filament organization in the actin cytoskeleton. Nat Methods 9, 185-188.

Zaidel-Bar, R., Itzkovitz, S., Ma'ayan, A., Iyengar, R., and Geiger, B. (2007). Functional atlas of the integrin adhesome. Nat Cell Biol 9, 858-867. 

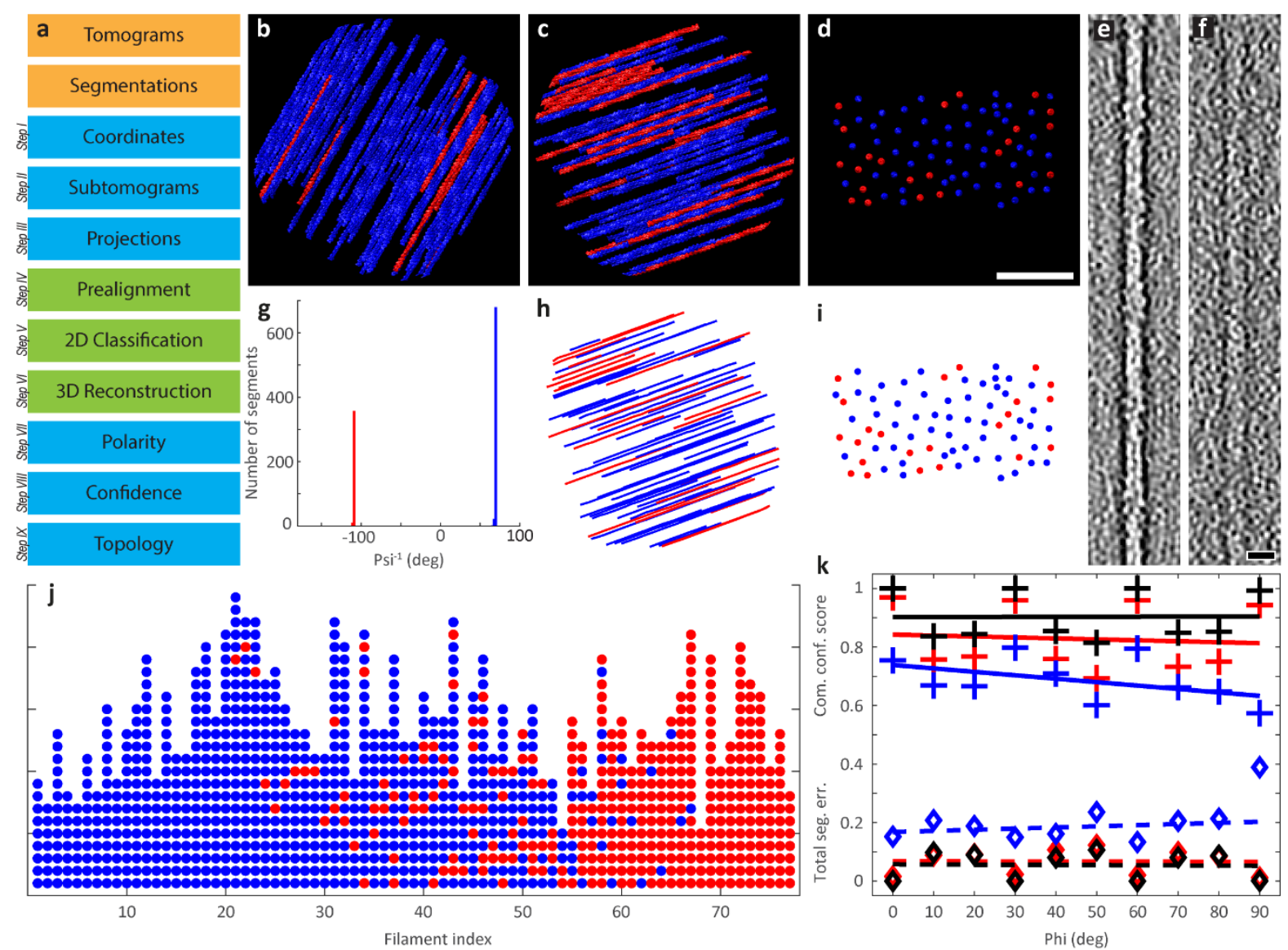

693

Figure 1 | Polarity determination of modelled actin bundles. (a) The workflow of APT

695

696

697

698

699

700

701

702

703

704

705

706

consists of consecutive modules. The orange modules symbolize the required input data. The

blue modules were implemented in MATLAB, and the green modules were executed in RELION. (b) Top view of one of the modelled actin bundles, which were used for the validation of APT. The bundles were rendered without noise for visualization purposes. Here the angle $\varphi$ between bundle and tilt-axis is $30^{\circ}$, and the fraction $\rho_{r}$ of actin filaments, that are oriented in opposite direction (red colored filaments) was set to 0.1. (c) Top view of an additional bundle with $\varphi$ increased to $70^{\circ}$, and $\rho_{r}$ to 0.3 . (d) The same bundle seen from the side with the viewing axis adjusted parallel to the filaments. The distance between the filaments is $10-37 \mathrm{~nm}$. Scale bar $100 \mathrm{~nm}$. (e) In order to quantify the impact of noise on the precision of APT, the bundles were modelled with defined SNRs. The depicted bandpass filtered slice of a filament was extracted from a bundle with $\mathrm{SNR}=0.001$. For comparison, the filament shown in $(\mathbf{f})$ originates from a bundle with $S N R=0.0001$. Scale bar $10 \mathrm{~nm}$. 
(g) The modelled bundles were processed with APT. The plot shows the resulting $\psi^{-1}$ histogram of the bundle displayed in (c). Segments in the blue peak mainly originate from blue filaments, and the opposite for the red peak. The filaments point with their plus-ends in opposite directions, therefore the peaks are separated by $180^{\circ}$. Since $\rho_{r}$ was set to 0.3 in this bundle, the number of segments in the red peak is $\approx 30 \%$ of the total number of segments. (h) The line plot shows the filament positions and polarities of the bundle, shown in (c), as recovered by APT. It was affected with a SNR $=0.001$ in this case, however, the match between original and recovered bundle (the side view is shown in (i), and can be compared to (d)) is almost error free. (j) Here the $\xi\left(F_{i}^{j}\right)$ vectors of the recovered bundle are depicted. Segments, that are originating from the same filament are plotted as columns of circles. The colour scheme reflects to which peak the segments were assigned in the $\psi^{-1}$ histogram, shown in $(\mathbf{g})$, and the $\xi\left(F_{i}^{j}\right)$ vectors are sorted according to their fraction of segments linked to the blue peak. In this representation of the filaments, segments with incorrect orientation determination appear as distortions of otherwise uniformly colored columns. (k) Three datasets of ten modelled actin bundles each, with SNRs of $0.01,0.001$, and 0.0001 , were produced, subsequently processed with APT, and finally the ccs values and errors in the polarity determination were analyzed.

723 Each cross marks the average of all ccs values in a bundle. The SNR can be identified by the 724 color scheme: black, $\mathrm{SNR}=0.01$, red, $\mathrm{SNR}=0.001$, and blue, $\mathrm{SNR}=0.0001$, respectively. A regression line was fitted for each SNR condition, and show that there is no trend to lower ccs values with increasing $\varphi$ for SNRs $\gtrsim 0.001$ (black and red lines). Thus, the missing wedge

727 induced anisotropy, which is proportional to $\varphi$, can be neglected for those SNR levels. However, for SNRs $\lesssim 0.0001$ a correlation can be detected (blue line). Next, we calculated the average total segment error for each bundle, plotted as diamond symbols. Respective 730 regression lines are delineated as dashed lines. Even for the markedly challenging 731 SNR $=0.0001$ the total segment error rarely exceeds $20 \%$. Because APT uses a majority 732 criterion for final polarity determination of a whole filament, segment errors $<50 \%$ can 733 be compensated. 

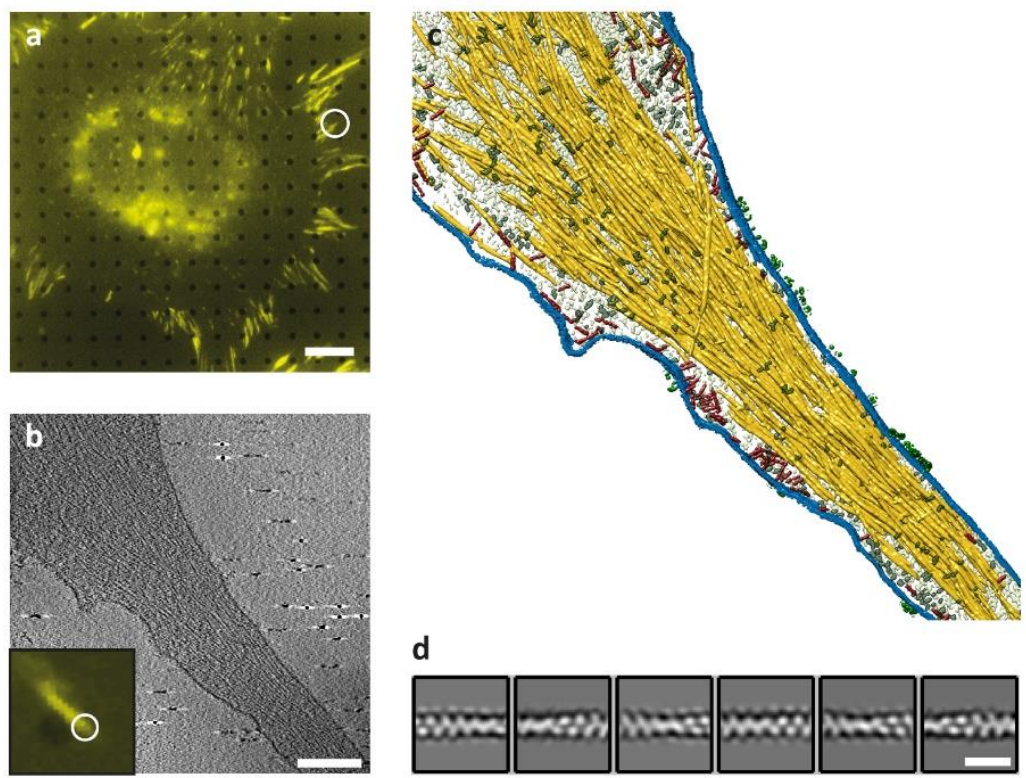

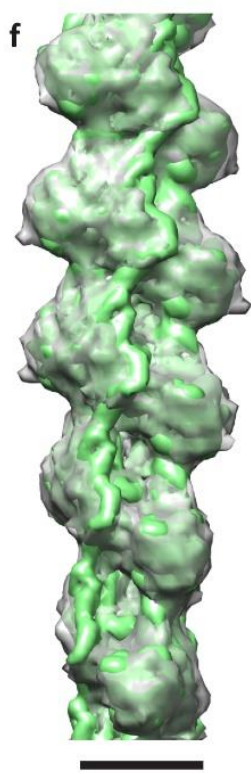

734

Figure 2 | Cryo-tomography of FAs and actin filament structure from inside cells.

Correlated microscopy combining fluorescence microscopy (a, b, inset) and cryo-ET (b) was used to identify FA sites. Scale bar in (a) $10 \mu \mathrm{m}$. (b) A $1.4 \mathrm{~nm}$ thick slice through a cryotomogram of a FA (a, b, white circles), shows individual actin filaments and plasma membrane.

Scale bar $200 \mathrm{~nm}$. (c) Surface rendering view of the FA site. Actin is depicted in yellow, membranes in blue, macromolecules in red and grey, and receptor densities in green. The segmentation of the actin filaments served as input for APT. (d) Class averages obtained by 2D classification of the extended dataset. Scale bar $18 \mathrm{~nm}$. (e) Structure of an actin filament at FAs (Supplementary Fig. 9), shown as grey isosurface. (f) The in-vitro structure EMD-6179 (Galkin et al., 2015) (green isosurface) was docked into the in-situ structure. Scale bar $5 \mathrm{~nm}$. 

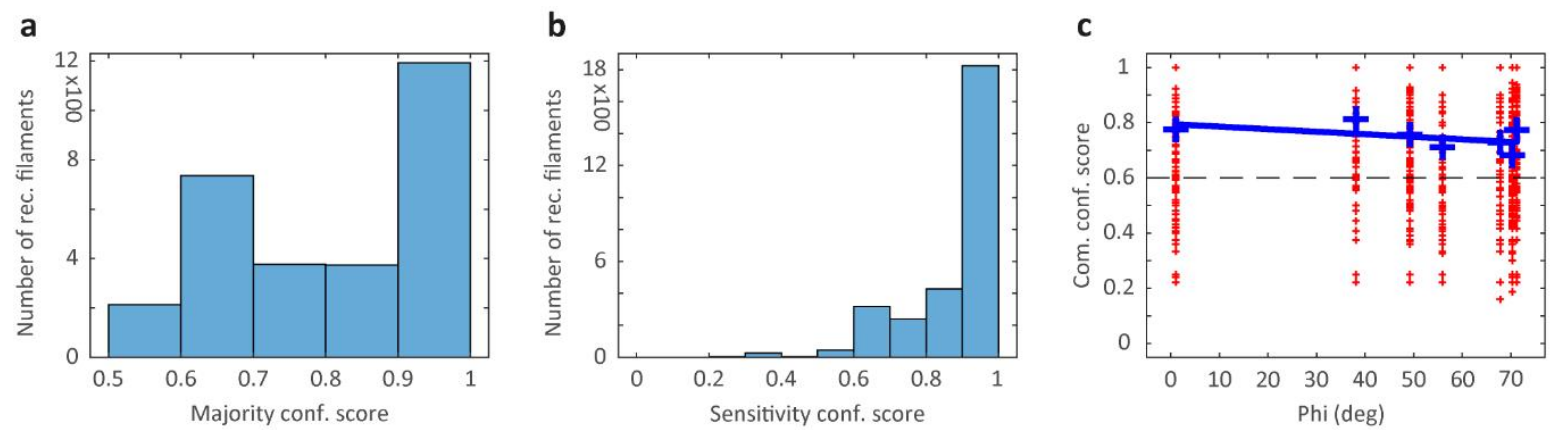

d

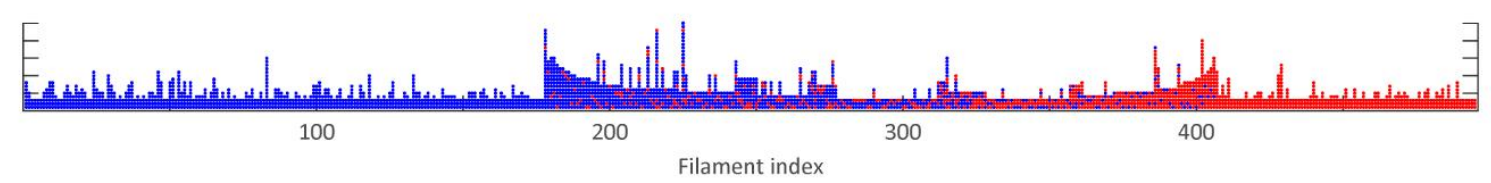

e

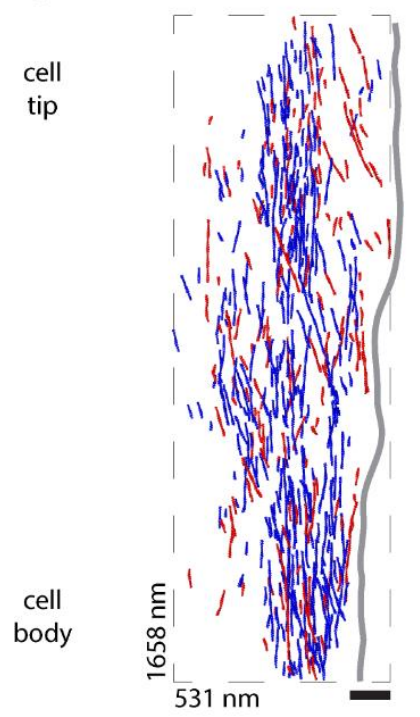

i
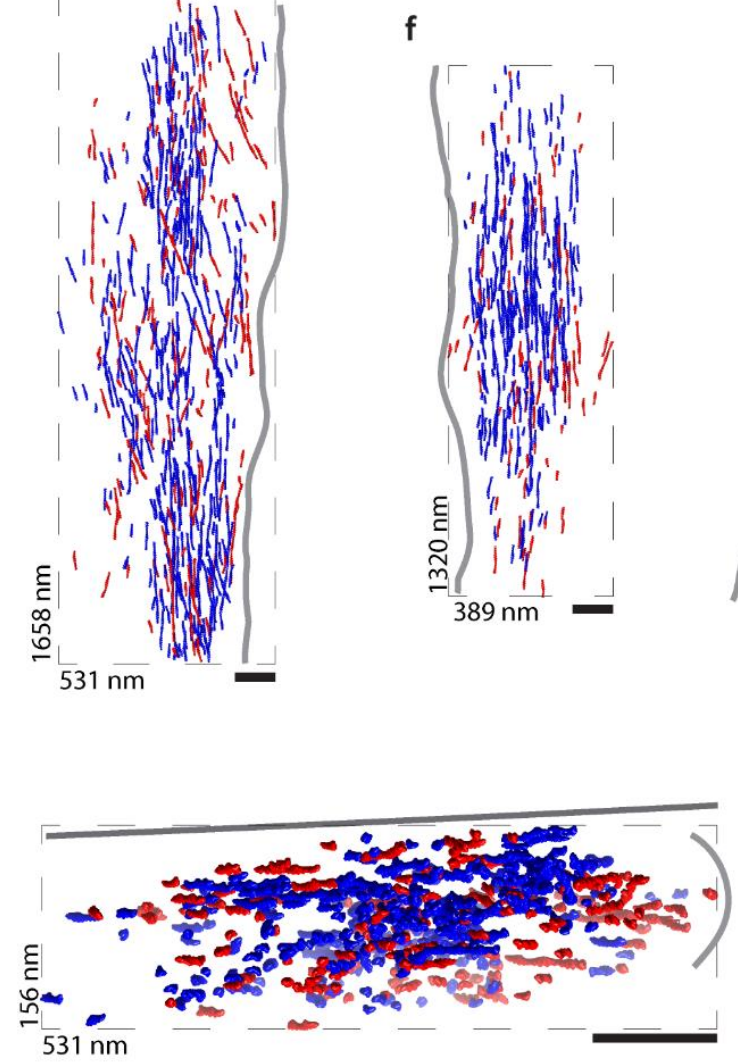

g

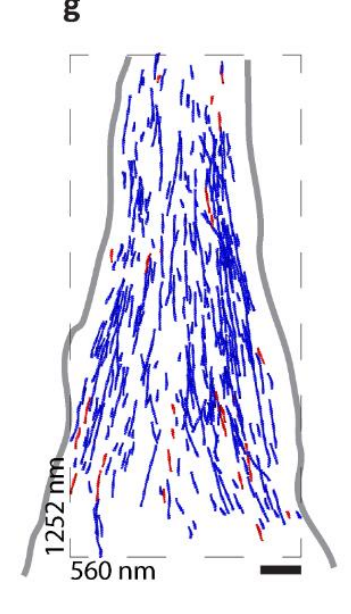

$\mathbf{k}$

748 Figure 3 | Architecture of actin bundles at FAs. (a) Histogram of the mcs values from 2893 reconstructed actin filaments, extracted from seven manually segmented cryo-tomograms acquired at FAs. (b) Histogram of the respective scs values. (c) Plot of the respective ccs

751 values. Each red cross marks the ccs of one filament, and the scores are plotted as a function 
752 of the bundle orientation $\varphi$. The blue crosses indicate the average of all ccs values that are

753 originating from the same actin bundle, and a regression line was fitted to these values (blue 754 line). Only filaments reaching a ccs $\geq 0.6$ (dashed line) were accepted, therefore 2146 755 reconstructed actin filaments were used for subsequent bundle visualization and topology 756 analysis. (d) Visualization of the resulting $\xi\left(F_{i}^{j}\right)$ vectors of a bundle that was recorded at a 757 proximal FA region. Segments, that are originating from the same filament are plotted as 758 columns of circles. Blue segments are oriented with their plus-ends towards the cell tip, and 759 red segments point in the direction of the cell body. In this bundle 495 filaments with an average 760 length of 7.1 segments were analysed. Approximately $2 / 3$ of the segments are directed 761 towards the cell tip. (e-h) Top views on the architecture and polarity distribution of four actin 762 bundles, recorded at proximal $(\mathbf{e , f})$ and distal $(\mathbf{g}, \mathbf{h})$ FA regions. Blue actin filaments are 763 oriented with their plus-ends towards the cell tip, and red filaments point in the direction of the 764 cell body. Proximal FA bundles are characterized by a tendentially mixed polarity distribution, 765 and distal FA bundles converge to a predominantly uniform polarity distribution. (i,j) Side views on the bundles shown in (e,h), respectively. (i) Filaments, which are directed towards the cell body (red filaments), are found with a higher probability at outer regions of proximal FA actin bundles. This relation is displayed in (k) as well, showing the long axis of bundle (e). In all

769 visualizations (e-k), actin filaments were rendered from EMD-6179 (Galkin et al., 2015), the 770 dashed rectangles are bounding boxes surrounding the actin bundles. Scale bars $100 \mathrm{~nm}$. 771 Additionally, positions of plasma membrane are suggested by light grey lines, and support 772 planes by dark grey lines. 


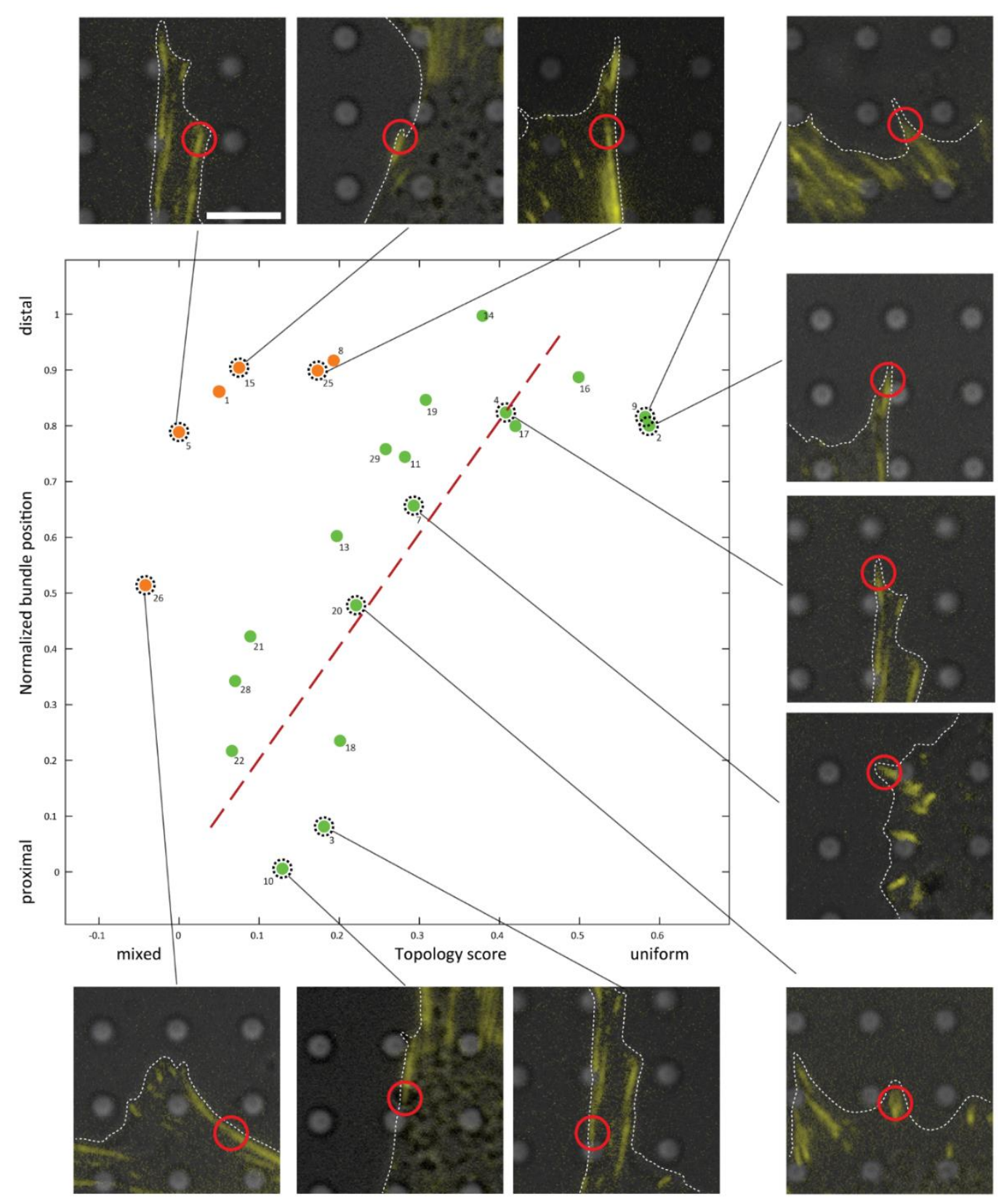

776 Figure 4 | The distribution of actin polarity at FAs. Plot of topology score versus normalized

777 bundle position along a FA. The green datapoints (fitted by the red dashed regression line)

778 suggest a correlation between these two parameters. The polarity distribution along FAs

779 transitions smoothly from mixed to uniform. However, in case that the distal region of a FA

780 does not coincide with a pronounced cell protrusion, the polarity distribution exhibits a

781 tendentially mixed character (orange datapoints). Some datapoints in the plot are

782 accompanied by their fluorescence microscopy signal. The plasma membrane is outlined by

783 white dashed lines, and the tomogram position is indicated by a red circle. Scale bar $5 \mu \mathrm{m}$. 


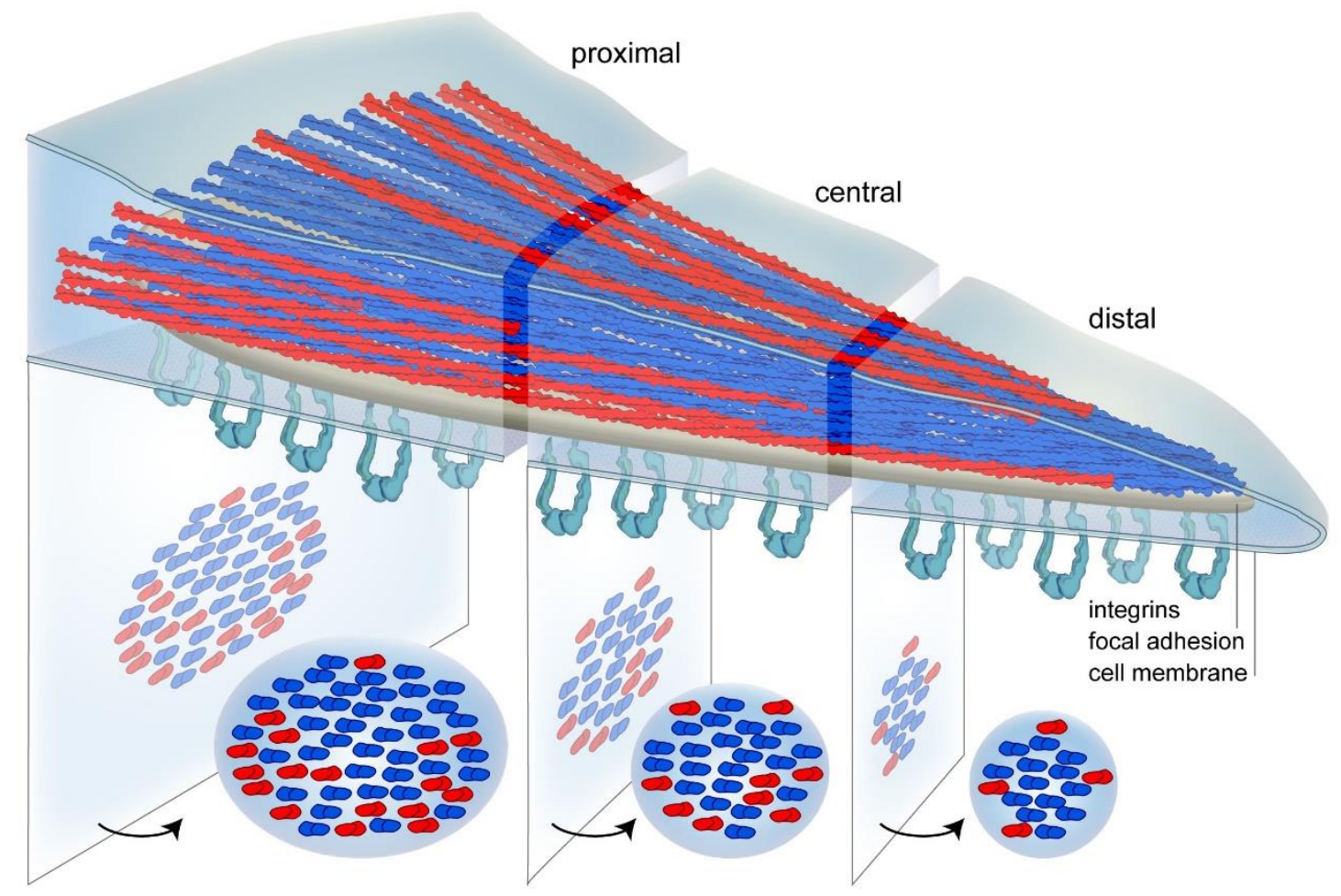

785 Figure 5 | A model for actin polarity at FAs. Actin bundles within FAs are characterized by 786 a mixed polarity distribution at proximal regions. However, there is a smooth transition towards a uniform polarity distribution at distal regions. Actin filaments, which are directed with their plus-ends towards the cell body (red), are arranged around a central core of filaments pointing

789 towards the cell periphery (blue). 